Präv Gesundheitsf 2023 · 18:1-9

https://doi.org/10.1007/s11553-021-00924-y

Eingegangen: 10. September 2021

Angenommen: 18. November 2021

Online publiziert: 14. Dezember 2021

(c) Der/die Autor(en) 2021

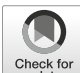

Check for
updates

Gerhard Meyer (D) - Tobias Hayer(D)

Studiengang Psychologie, Universität Bremen, Bremen, Deutschland

\title{
Schadensbegrenzung beim Glücksspiel
}

\section{Eine Aufgabe für die öffentliche Gesundheitsfürsorge}

Sofortlotterien, Keno oder Eurojackpot) und erhöhte die Spielanreize über die Einbindung von Jackpots. Illegale Angebote von Sportwetten in Wettbüros und im Internet tolerierte der Staat jahrelang, um sie dann mit dem ab dem 01.01.2020 in Kraft getretenen Dritten Glücksspieländerungsstaatsvertrag (GlüStV) in Form einer Übergangslösung in die Legalität zu überführen. Am 01.07.2021 fand dieser Entwicklungstrend mit dem neuen GlüStV 2021 seinen vorläufigen Höhepunkt, da bundesweit ein Multikonzessionsmodell für virtuelle Automatenspiele, Online-Poker und Online-Casinos etabliert wurde. Diese Komplettöffnung des Online-Markts auch für Privatunternehmen dürfte die (wahrgenommene) Verfügbarkeit und Griffnähe von Glücksspielen abermals deutlich erhöhen.

Diese Marktausweitung lässt sich auch durch ökonomische Zahlen dokumentieren: So sind die Umsätze (Spieleinsätze) auf dem legalen Glücksspielmarkt in Deutschland bis 2018 kontinuierlich gestiegen, von $2,9 \mathrm{Mrd}$. $€$ in 1974 über $27,4 \mathrm{Mrd} . €$ in 2002 und $36,8 \mathrm{Mrd}$. $€$ in 2012 auf 45,8 Mrd. $€$ in 2018 (in 2019 ist gegenüber dem Vorjahr ein leichter Rückgang um 3,5\% feststellbar [32]). Gleiches gilt für die Bruttospielerträge (Differenz von Spieleinsätzen und Spielgewinnen) der legalen Anbieter, die zuletzt ein Volumen von $11,5 \mathrm{Mrd}$. $€$ erreichten (illegaler Markt: 2,2 Mrd. €). Der Staat partizipierte in 2019 mit (Steuer)einnahmen von insgesamt 5,4 Mrd. $€$. Demgegenüber zeichnen Populationsstudien ein differenzierteres Bild: Nach repräsentativen Erhebungen in der Be- völkerung, die die Bundeszentrale für gesundheitliche Aufklärung (BZgA) seit 2007 in 2-jährigem Abstand durchführt, gaben in 2007 86,5\% der Befragten an, sich im Laufe des Lebens schon einmal an irgendeinem Glücksspiel beteiligt $\mathrm{zu}$ haben [7]. Bezogen auf die letzten 12 Monate lag diese Kennzahl bei $55 \%$. Die Teilnahmeprävalenzen erweisen sich allerdings als rückläufig: In 2019 wurden Lebenszeit- und 12-Monats-Werte von 75,3\% bzw. 37,7\% ermittelt. Daneben finden sich empirische Befunde zum Ausmaß glücksspielbezogener Probleme von inzwischen 11 repräsentativen Bevölkerungsstudien, die über die Jahre hinweg keine signifikanten Veränderungen zeigen (vgl. im Überblick mit [32]). Die aktuellste Studie der BZgA [7] beziffert für 2019 den Anteil der problematischen Spieler*innen (im Sinne einer missbräuchlichen Nutzung des Glücksspiels) in der 16- bis 70-jährigen Bevölkerung mit 0,39\% (ca. 229.000 Personen) und den der pathologischen Spieler*innen mit $0,34 \%$ (ca. 200.000 Personen). Forschungsergebnisse aus anderen Ländern verweisen in diesem Zusammenhang auf ähnliche Entwicklungen, wie etwa in den Niederlanden [18], Schweden [5] oder Neuseeland [1]. Mit zunehmender Verfügbarkeit von Glücksspielen stiegen zunächst die Spielteilnahme und Geldausgaben in der Bevölkerung sowie die Prävalenzraten glücksspielbezogener Störungen an. Trotz eines weiter wachsenden Angebots ging die Spielteilnahme im letzten Jahrzehnt zurück, während sich die Prävalenzraten auf einem Plateau einpendelten. Initial ansteigende und später stabilisierende Prävalenzsukzessiv neue Lotterieprodukte (u.a. 
raten ermittelten Williams et al. [48] ebenfalls für die USA, Kanada und Australien.

Nach den zunehmenden Erkenntnissen zu den mit Glücksspielen assoziierten Suchtgefahren sowie öffentlicher Kritik an den Wachstumsstrategien der Anbieter und an unzureichender staatlicher Reglementierung erfolgte als Reaktion - zunächst v.a. im angelsächsischen Sprachraum - die Einführung von Maßnahmen des sog. „Responsible Gambling“. Hierbei handelt es sich um eine Reihe von gemeinsamen Initiativen der Glücksspielanbieter, Regierungen und Kontrollgremien zur Gewährung der Integrität und Fairness der Geschäftstätigkeit der Anbieter zum einen und zur generellen Förderung des Bewusstseins für das Gefährdungs- und Suchtpotenzial von Glücksspielen zum anderen. Ein erster Ordnungsrahmen aus dem Bereich der Wissenschaft, der weltweit zum Einsatz gekommen ist, stammt von Blaszczynski et al. [8] und wird als „Reno-Modell“ bezeichnet. Auf diesem Ansatz basierende politische, industrielle und gewerbliche Handlungsstrategien sind in den letzten Jahren jedoch verstärkt in die Kritik geraten, da ihr Fokus primär auf der individuellen Verantwortung der Spieler ${ }^{*}$ innen liegt. Zudem fordert das Reno-Modell vorrangig die Umsetzung von denjenigen Maßnahmen ein, die mit eher geringer suchtpräventiver Wirksamkeit einhergehen (wie z.B. Aufklärung der Konsument*innen dar; für die bestenfalls mäßige Effektivität diesbezüglich s. [25]). Als Alternative wird inzwischen - in Anlehnung an den Umgang mit Suchtmitteln wie Alkohol und Tabak - auf ein Konzept für die öffentliche Gesundheitsfürsorge (sog. Public-Health-Ansatz) verwiesen, dessen Implementierung sektorenübergreifende Maßnahmen und die Beteiligung der gesamten Gesellschaft erfordert (z. B. [3, 13, 47]).

Vor diesem Hintergrund stellt sich die Frage, wie die beiden Ansätze grundsätzlich einzuordnen und aus gesundheitswissenschaftlicher Perspektive zu bewerten sind. Mit anderen Worten: Ist das Konzept eines verantwortungsbewussten Umgangs mit Glücksspielen, das sich primär an Anbieter und Spielteilnehmer ${ }^{*}$ innen richtet oder der konzeptuelle
Ansatz der öffentlichen Gesundheitsfürsorge, der die gesamte Gesellschaft in das Blickfeld rückt, eher geeignet, dem Ziel einer effektiven Schadensbegrenzung gerecht $\mathrm{zu}$ werden? Unabhängig von der Beantwortung dieser Fragestellung intendiert der vorliegende Überblicksbeitrag zugleich, den öffentlichen Diskurs um eine evidenzgestützte Abwehr von glücksspielbezogenen Suchtgefahren hierzulande zu stimulieren und um weitere zentrale Aspekte zu ergänzen.

\section{Das Reno-Modell: ein konzeptueller Handlungs- rahmen für einen verantwortungsbewussten Umgang mit Glücksspielen}

Blaszczynski et al. [8] haben in ihrem Reno-Modell erstmals einen strategischen Handlungsrahmen für einen verantwortungsbewussten Umgang mit Glücksspielen formuliert, der in den Folgejahren weiterentwickelt und aktualisiert wurde (z.B. [27, 41]). Die Grundannahmen dieses Ansatzes lauten (vgl. [19]):

- Glücksspiel stellt eine legale, regulierte Form der Unterhaltung und Freizeitgestaltung dar.

- Die Entscheidung zur Spielteilnahme liegt in letzter Konsequenz beim Individuum. Um diese Entscheidung wohlüberlegt treffen zu können, müssen die Spieler*innen über die Funktionsweise der Spielformen und die potenziellen Konsequenzen ihres Handelns bestmöglich informiert werden.

- Glücksspiele können einer kleinen Anzahl von Spieler*innen prinzipiell schaden. Die als „ResponsibleGambling-Maßnahmen“ bezeichneten Interventionen sollen sich in erster Linie an Hochrisikopopulationen bzw. Problemspieler ${ }^{\star}$ innen und damit vulnerable Personengruppen richten.

- Maßnahmen des „Responsible Gambling“ sind sorgfältig darauf hin zu überprüfen, ob sie das Vergnügen von Freizeitspieler*innen minimieren.

- Der soziale Nutzen legaler Spielangebote übersteigt die sozialen Kosten.
- Die wissenschaftliche Forschung kann und sollte Strategien zur Schadensbegrenzung leiten.

- Zentrale Stakeholder des „Responsible Gambling" verfolgen ähnliche Ziele und müssen daher zusammenarbeiten.

- Glücksspielanbieter dürfen gefährdete Personen nicht wissentlich ausbeuten.

Dieser konzeptuelle Handlungsrahmen, ursprünglich als Diskussionsgrundlage einer Forschungsagenda für effektive Maßnahmen des „Responsible Gambling" gedacht, fand schnell weltweite Zustimmung v.a. bei Regierungen, Regulierungsbehörden und der Glücksspielindustrie. Abgeleitet und umgesetzt wurden Präventionsmaßnahmen, die typischerweise drei Kategorien zuzuordnen sind (vgl. [19]):

1. Aufklärung der Öffentlichkeit (v.a. Informationen über die Funktionsweise der Spielangebote, Tipps für einen verantwortungsvollen Umgang mit Glücksspielen sowie Hinweise auf Erkennungsmerkmale problematischen Spielverhaltens),

2. Hinweise auf Beratungsangebote und Support-Leistungen (wie TelefonHelplines, Beratungsangebote vor Ort oder Selbstsperren),

3. Etablierung eines verantwortungsbewussten Umfelds (z. B. mittels eines Verhaltenskodex, der Sensibilisierung über Mitarbeiterschulungen, selbstbestimmter Werberichtlinien und Akkreditierung der ResponsibleGambling-Maßnahmen).

Die Praxisimplikationen erweisen sich aufgrund der mangelhaften Evidenzbasierung jedoch als angreifbar. Zum einen lassen umfassende Bestandsaufnahmen zur Wirksamkeit verschiedener Maßnahmen des Jugend- und Spielerschutzes (z.B. [25, 49]) die Schlussfolgerung zu, dass der suchtpräventive Nutzen der im Reno-Modell vorgeschlagenen Handlungsansätze generell als eher gering einzuschätzen ist. Zum anderen beklagen kritische Stellungnahmen die Fokussierung auf verhaltenspräventive Maßnahmen, die die individuelle Verantwortung der Spieler ${ }^{*}$ innen in übermäßi- 
ger Weise betonen. Zugleich werden verhältnispräventive Maßnahmen, wie die Begrenzung der generellen Verfügbarkeit oder Eingriffe in Spielstrukturen und das Umfeld, weitgehend vernachlässigt (z. B. [19, 20, 37]). In der Fortschreibung des Reno-Modells [12] werfen die Autoren immerhin Forschungsfragen auf, die auf den Einfluss von wesentlichen Gestaltungsmerkmalen des „Game Design“, wie Einsatz- und Gewinnhöhe bzw. Spieldauer, sowie der Verfügbarkeit von Glücksspielen abzielen, ohne jedoch auf die gesamte Bandbreite der vorhandenen Evidenz einzugehen. Aufgrund des Mangels an methodisch hochwertigen Evaluationsstudien lassen sich zum jetzigen Zeitpunkt tatsächlich zwar kaum belastbare Aussagen zur Wirksamkeit von Veränderungen bestimmter GameDesign-Elemente ableiten [49]. Unter theoretischen Gesichtspunkten dürften aber primär von einer Verlangsamung der Spielgeschwindigkeit Positiveffekte im Sinne der Gefahrenabwehr ausgehen. Empirische Befunde einer Metaanalyse bestätigen in diesem Kontext, dass die Teilnahme an kontinuierlichen Spielformen - damit sind Spielangebote mit hoher Ereignisfrequenz gemeint - als besonders gefährlich gilt [6]. Darüber hinaus liegen empirische Belege für die Effektivität von Verfügbarkeitsbegrenzungen, vergleichbar mit den Erkenntnissen bei substanzbezogenen Störungen, auch im Glücksspielbereich vor [34].

Eine weitere umstrittene Grundannahme des Reno-Modells besteht in der propagierten ähnlichen Zielsetzung aller wichtigen Stakeholder. Entgegen dieser Prämisse erweisen sich die Ziele in der Praxis allerdings oftmals als unvereinbar: So bildet z. B. die Gewinnmaximierung die primäre Intention der Glücksspielanbieter, während die Schadensminimierung, die zwangsläufig mit Ertragsrückgängen einhergeht, das Hauptanliegen des Verbraucherschutzes. Interessenkonflikte auf Seiten der Anbieter lassen sich daher nicht wegdiskutieren (für zahlreiche Belege s. [31]). Infolgedessen dürfte der Appell an die Anbieter, gefährdete Spieler*innen nicht wissentlich auszubeuten, kaum hinreichend sein. Weltweit ist für verschiedene

Präv Gesundheitsf 2023 · 18:1-9 https://doi.org/10.1007/s11553-021-00924-y

(c) Der/die Autor(en) 2021

\section{G. Meyer · T. Hayer}

\section{Schadensbegrenzung beim Glücksspiel. Eine Aufgabe für die öffentliche Gesundheitsfürsorge}

\section{Zusammenfassung}

Hintergrund. Die Verfügbarkeit von Glücksspielen ist in Deutschland seit Mitte der 1970er-Jahre stark gestiegen. Als Reaktion auf potenzielle Folgeschäden dieses Entwicklungstrends wurden von industrieller und staatlicher Seite Maßnahmen eines verantwortungsbewussten Umgangs mit Glücksspielen (Responsible Gambling) ergriffen. Kritische Analysen dieses Präventionsansatzes verweisen alternativ auf ein Konzept der öffentlichen Gesundheitsfürsorge (Public Health). Fragestellung. Vor dem Hintergrund des neuen Glücksspielstaatsvertrags, der am 01. Juli 2021 in Kraft getreten ist, stellt sich die Frage, wie die beiden Ansätze einzuordnen und hinsichtlich einer effektiven Schadensbegrenzung zu bewerten sind. Methode. Im Zuge eines narrativen Reviews erfahren die Kernaussagen dieser beiden Ansätze eine kritische Analyse.

Ergebnisse. Das Konzept des verantwortungsbewussten Umgangs mit Glücksspielen fokussiert primär auf die individuelle Verantwortung und Vulnerabilität der Spieler*innen.
Verhältnispräventive Maßnahmen, wie die Reduktion der Verfügbarkeit, Beschränkungen der Spielanreize durch Eingriffe in die Spielstruktur und Werberestriktionen, die Interessenkonflikte der Anbieter hervorrufen, fehlen weitgehend. Der interdisziplinäre Public-Health-Ansatz ist breiter ausgerichtet und berücksichtigt neben individuellen Risikofaktoren zielgenauer soziale, umweltbezogene und wirtschaftliche Faktoren, die zusammen mit der Identifizierung schädlicher Eigenschaften des Produkts "Glücksspiel" zu einer umfassenden Präventionsstrategie führen.

Schlussfolgerung. Neue Erkenntnisse in den Bereichen Forschung und Praxis sowie die zunehmende Akzeptanz evidenzbasierter Public-Health-Strategien wecken Hoffnungen, dass dieser Weg zukünftig auch in Deutschland beschritten wird.

Schlüsselwörter

Glücksspielbezogene Probleme · Glücksspielsucht - Prävention · Public-Health-Strategie . Spielerschutz

\section{Harm reduction in gambling. A public health task}

\section{Abstract}

Background. The availability of gambling in Germany has increased sharply since the mid-1970s. In response to potential negative effects of this developmental trend, measures of responsible gambling have been taken by industry and government. Critical analyses of this prevention approach point alternatively to the concept of public health.

Objective. Against the background of the new State Treaty on Gambling, which came into force on 01 July 2021, the question arises as to how the two approaches are to be classified and evaluated with regard to effective harm reduction.

Methods. In the course of a narrative review, the core statements of these two approaches are critically analyzed.

Results. The concept of responsible gambling focuses primarily on the individual responsibility and vulnerability of gamblers. Structural preventive measures, such as the reduction of availability, manipulation of gambling incentives by intervening in the structure of gambling forms, and advertising restrictions that cause conflicts of interest for providers, are largely missing. The interdisciplinary public health approach however is broader in scope and, in addition to individual risk factors, takes more targeted account of social, environmental and economic factors, which, together with the identification of harmful characteristics of the product "gambling", lead to a more comprehensive prevention strategy. Conclusion. New findings in the areas of research and practice as well as the increasing acceptance of evidence-based public health strategies raise hopes that this path will also be followed in Germany in the future.

\section{Keywords}

Problem gambling - Gambling addiction . Prevention · Public health strategy · Gambler protection 
Spielformen (insbesondere Spielautomaten und Tischspiele in Spielbanken) nachweisbar, dass der von Problemspieler*innen generierte Umsatzanteil unverhältnismäßig hoch ausfällt und bis zu $76 \%$ beträgt [17]. Stehen verschiedene Glücksspielunternehmen zudem innerhalb eines Marktsegments in direkter Konkurrenz zueinander, liegt es nahe, dass effektiver Spielerschutz sogar einen Wettbewerbsnachteil bedeuten kann. Als empirischer Beleg für die mangelhafte Compliance der Anbieter dient exemplarisch die Evaluation des Sperrsystems der Spielhallen im Bundesland Hessen [23]. Obwohl die Spielhallenbetreiber gesetzlich verpflichtet sind, erkennbar süchtige Spieler*innen von der Spielteilnahme auszuschließen, belief sich der Anteil der Sperren durch das Personal der Spielhallen auf $<1 \%$ (99\% beruhten auf Selbstsperren der Betroffenen). Auch auf Anzeichen von (simulierten) Spielproblemen zeigte das Personal der Spielhallen kaum angemessene Reaktionen. Konsistent hierzu berichtete nur etwa $1 \%$ der Spieler ${ }^{\star}$ innen aus Behandlungseinrichtungen, dass sie vom Personal auf ihr problematisches Spielverhalten angesprochen und in das Hilfesystem vermittelt wurden [17]. Ähnliche Befunde stammen aus Australien [38]: Statt präventiv zu agieren, wurde sogar häufig eine Fortsetzung des fehlangepassten Spielverhaltens gefördert. Schließlich scheinen Maßnahmen des Spielerschutzes, die von Anbieterseite lediglich vorgehalten werden bzw. ausschließlich auf einer freiwilligen Nutzung basieren, bestenfalls mäßige Wirksamkeit zu entfachen. Beispielhaft hierfür stehen Pre-Commitment-Systeme, die Begrenzungen der Spielzeit, des Einsatzes, möglicher Gewinne und/oder Verluste vorsehen. Ein wesentliches Manko bei Limitierungsprogrammen, die auf Freiwilligkeit setzen, besteht in der geringen Inanspruchnahmerate. Entsprechend erweisen sich (gesetzlich) verbindliche Systeme gegenüber freiwilligen Programmen unter suchtpräventiven Gesichtspunkten als überlegen [15].

Die Forderung nach einer Kooperation verschiedener Stakeholder hat außerdem einen kontroversen Diskurs um industriegeförderte Forschungsprojekte ausgelöst. Bei derartigen Geschäftsbeziehungen besteht die grundsätzliche Gefahr von Veröffentlichungen, die verzerrende Ergebnisse im Sinne der Glücksspielanbieter beinhalten („Publication Bias"). Aber auch subtilere Einflussnahmen, wie die gemeinsame Auswahl ablenkender bzw. weitgehend irrelevanter Forschungsfragen oder die Veröffentlichung eher banaler Forschungsbefunde, zählen zu diesem Gefahrenspektrum [11]. In den Bereichen Alkohol, Tabak und Pharmazeutika sind entsprechende Nachweise des Einflusses der Industrie auf die Forschung schon seit längerem bekannt [2, 44]. Shaffer et al. [40] halten indessen eine transparente Offenlegung von Finanzierungsquellen der Forschenden und Projekte für ausreichend, um die Öffentlichkeit über den potenziellen Einflussfaktor der Voreingenommenheit $\mathrm{zu}$ informieren. Gleichzeitig verweisen sie auf die Notwendigkeit des Zugangs $\mathrm{zu}$ echten Glücksspieldaten und Spielteilnehmer*innen in natürlichen Spielsituationen, was nur mit Unterstützung der Industrie möglich erscheint. Vor diesem Hintergrund kommt es wenig überraschend, dass die Arbeitsgruppe um Shaffer [42] selbst in einer Analyse von insgesamt 720 Primärstudien keinen „Funding Bias“ feststellen konnte: So scheint die Finanzierungsquelle weder die methodologische Güte noch die Outcomes der Primärstudien zu beeinflussen. Eine Replikation dieses Befunds von unabhängiger Seite steht allerdings noch aus. Der Vollständigkeit halber sei noch hinzugefügt, dass staatliche Einrichtungen ebenfalls bestimmte Interessen verfolgen (vgl. [19, 20]). Unter anderem sind Regierungen selbst häufig Nutznießer des Glücksspiels, aber auch dem Lobbyismus der Industrie ausgesetzt, was in beiden Fällen zu einer Verhinderung effektiven Spielerschutzes führen kann.

Auch in Deutschland haben sich einzelne Personen aus der Wissenschaft und Suchthilfe einer Initiative von Vertreter*innen und Berater*innen der Glücksspielindustrie mit dem Namen „Düsseldorfer Kreis“ angeschlossen, um Vorschläge für eine am Verbraucherschutz orientierte Regulierung staatli- cher und gemeinnütziger gewerblicher Glücksspielangebote zu etablieren. ${ }^{1}$ Wie die Repräsentanten des Reno-Modells setzen sie in erster Linie auf die Verantwortung der Spieler*innen, Regulierungsstellen und Glücksspielbetreiber für einen gesetzlich konsensfähigen und wirksamen Verbraucherschutz. Bei näherer Betrachtung dieses Konzepts, eines Positionspapiers zur Glücksspielwerbung und der Publikationen von Mitgliedern der Initiative ist zu erkennen, dass der Düsseldorfer Kreis vornehmlich den Interessen der Anbieter dient [30]. Zentrale Maßnahmen des Verbraucherbzw. Spielerschutzes, etwa die Reduktion der Verfügbarkeit des Suchtmittels „Glücksspiel“, werden unter Missachtung der vorliegenden Evidenz [34] als kaum effektiv dargestellt. Eine Differenzierung des Gefährdungspotenzials einzelner Spielformen und als Folge die Forderung nach Eingriffen in die Spielstrukturen finden ebenso wenig Beachtung. Zudem lehnt der Düsseldorfer Kreis aktuell spezifische Werbeeinschränkungen für einzelne Glücksspiele ab, da ihr präventiver Mehrwert wissenschaftlich nicht belegt sei. Hierbei verkennen die Mitglieder jedoch zum einen, dass Werbung für legale Suchtmittel (Alkohol, Tabak, Glücksspiel) immer auf die Gewinnung neuer Konsument*innen und deren dauerhafte Bindung sowie die Modellierung von Einstellungsmustern bzw. Verhaltensintentionen ausgerichtet ist und damit anerkannten gesundheitspolitischen Zielen widerspricht [24]. Zum anderen ignoriert diese Darstellung abermals zahlreiche empirische Forschungsbefunde, die die Beeinflussung v. a. vulnerabler Bevölkerungsgruppen, wie Heranwachsende oder Personen mit einem problematischen Spielverhalten, durch Werbeformate und -inhalte belegen [35].

Unabhängig davon stellt sich die Frage nach der grundsätzlichen Rolle der Glücksspielanbieter bei der Entwicklung und Umsetzung von Maßnahmen des Spielerschutzes. Unbestritten ist, dass gewisse Forschungsvorhaben nur in Kooperation mit Glücksspielanbietern funktio-

\footnotetext{
1 Für weitere Informationen s. https://www.
} duesseldorfer-kreis.de. 
nieren, z. B. indem (Spielverhaltens-)Daten für wissenschaftliche Zwecke zur Verfügung gestellt werden. Auf dieser Grundlage ließen sich in der Vergangenheit in bestimmten Domänen des „Responsible Gambling“ deutliche Fortschritte erzielen, etwa mit der Weiterentwicklung von Früherkennungssystemen sowohl beim Online- als auch beim Offline-Glücksspiel (vgl. [33]). Demgegenüber zeigen die bisherigen Erfahrungen aber auch, dass v. a. selbstverpflichtende Beschränkungen unter der Agenda der Suchtprävention bestenfalls mit mäßiger Wirksamkeit einhergegangen sind oder oftmals schlichtweg Alibifunktion besessen haben [31]. Eine aktuelle Blaupause hierfür stellt die sog. Qualitätsoffensive der gewerblichen Automatenbranche dar, die mit einer (ursprünglich freiwilligen) Zertifizierungspraxis und in der Konsequenz mit einem verbesserten Spielerschutz wirbt. Ob sich zertifizierte Spielhallen diesbezüglich tatsächlich nicht-zertifizierten Spielhallen als überlegen erweisen, muss aufgrund des Fehlens von empirischen Forschungsdaten indessen offen bleiben. Daneben zeigt der Bereich der Personalschulung lehrbuchhaft, wie aus Anbietersicht eine Win-Win-Situation geschaffen werden konnte, ohne den Spielerschutz substanziell zu verbessern: So haben sich in den letzten Jahren Privatunternehmen bzw. Gesellschaften mit unmittelbarer oder mittelbarer Nähe zur Glücksspielindustrie konstituiert, die zur Erfüllung der gesetzlichen Anforderungen kostenpflichtige Personalschulungen anbieten. Während beide Instanzen von diesen Geschäftsbeziehungen profitieren, sind suchtpräventive Effekte (etwa in einer Erhöhung der Anzahl anbieterinitiierter Fremdsperren) nicht $\mathrm{zu}$ belegen [23]. Ähnliche Business-to-BusinessGeschäftsbeziehungen mit unsicheren Effekten für den Spielerschutz bahnen sich im Übrigen bei der Spielsuchtsuchtfrüherkennung im Internet an. Auch hier drängen Unternehmen mit eindeutig wirtschaftlichen Interessen auf den deutschen Markt, um die eigenen Früherkennungsprodukte zu bewerben. Zusammenfassend sollte sich die Aufgabe der Glücksspielanbieter somit in erster Linie darauf beschränken, die ge- setzlich vorgegebenen Maßnahmen des Spielerschutzes vor Ort konsequent umzusetzen. Das schließt zwar per se nicht aus, dass einzelne von der Anbieterseite implementierte Interventionen im Sinne des „Responsible Gambling“ Teil eines umfassenderen Präventionsansatzes bilden könnten. Zwingend erforderlich hierfür sind aber wissenschaftliche Wirksamkeitsnachweise und eine engmaschige wie konsequente Kontrolle der Umsetzung durch eine Aufsichtsbehörde.

\section{Der interdisziplinäre Ansatz der öffentlichen Gesundheitsfürsorge}

Nach der weltweiten Implementierung von Maßnahmen des „Responsible Gambling“ durch die Glücksspielindustrie und jeweiligen Regierungen sowie der wachsenden Kritik daran wurde in einigen Ländern eine alternative Strategie diskutiert und bereits umgesetzt: das Konzept der öffentlichen Gesundheitsfürsorge (Public Health). Im Zentrum des Public-Health-Ansatzes steht der Gedanke, die Lebens- und Arbeitsbedingungen für alle Menschen derart $\mathrm{zu}$ gestalten, dass sie Gesundheit fördern bzw. vor Krankheiten schützen [39]. Bereits Ende der 1990er-Jahre plädierten Korn und Shaffer [26] dafür, das Angebot von Glücksspielen aus der Perspektive der öffentlichen Gesundheit zu betrachten. Der umfassende Anspruch der Perspektive betrachtet das Individuum innerhalb eines sozialen Umfelds, erforscht den Einfluss von familiären, gesellschaftlichen und kulturellen Werten auf das individuelle Verhalten, hinterfragt politische und wirtschaftliche Entscheidungen und führt auf diesem Weg zu vielfältigen Strategien und Ansatzpunkten für Interventionen. Gleichzeitig werden sowohl die Vor- als auch die Nachteile von Glücksspielen in Betracht gezogen.

Die offizielle Anerkennung des Glücksspiels als Problem der öffentlichen Gesundheit ging anschließend auf internationaler Ebene allerdings nur sehr schleppend voran. Eine Ausnahme bildet Neuseeland, dort wurde dieses Paradigma bereits in 2003 im verab- schiedeten "Gambling Act“ umgesetzt [36]. Das Gesetz beinhaltet, dass alle Strategien auf Schadensbegrenzung und Prävention, Förderung der öffentlichen Gesundheit, Behandlungsangebote für Problemspieler*innen und Angehörige, unabhängige glücksspielbezogene Forschung und Evaluation der Maßnahmen ausgerichtet sein müssen. In vielen anderen Ländern wird die glücksspielbezogene Schadensbegrenzung zwar inzwischen als Aufgabe der öffentlichen Gesundheitsfürsorge angesehen, es fehlt aber an der entsprechenden Einbindung in die Gesetzgebung. Daran mangelt es auch in Großbritannien, einem Land, das sich lange Zeit durch eine sehr liberale Gesetzgebung im Glücksspielbereich auszeichnete. Wardle et al. [47] fordern hier mehr als die bloße Feststellung der Problematik: Notwendig sei vielmehr auf politischer Ebene die Entwicklung und Umsetzung einer anerkannten und nachhaltig finanzierten Strategie. In diesem Zusammenhang sind insbesondere zwei empirische Argumente von Relevanz: Zum einen ist die Subgruppe der Geschädigten weitaus größer als die Subgruppe der Problemspieler*innen, da ebenso auf niedrigem Risikoniveau des Spielverhaltens ein Verlust an Lebensqualität verzeichnet werden kann. Zum anderen fallen die glücksspielbedingten sozialen Kosten deutlich höher aus als die staatlichen Steuereinnahmen (vgl. exemplarisch mit Befunden aus Australien: [9, 10]). Hinzu kommt, dass glücksspielbezogene Schäden in der Bevölkerung ungleich verteilt sind und bestimmte Personengruppen eine vergleichsweise große Krankheitslast tragen (vgl. [21]). Daneben lassen sich Spielstätten in der Regel überzufällig häufig in Gebieten vorfinden, die als sozialstrukturell unterprivilegiert gelten. Infolgedessen überraschen Befunde aus Baden-Württemberg nicht, nach denen ein deutlicher Zusammenhang zwischen der Dichte an Geldspielautomaten und dem Anteil an arbeitslosen Personen auf Gemeindeebene besteht [50]. Schließlich erleben nicht nur die Betroffenen selbst, sondern auch ihr soziales $\mathrm{Na}$ humfeld wie die Familie mannigfaltige Belastungen. Dazu zählen in erster Linie verschiedenartige gesundheitliche 


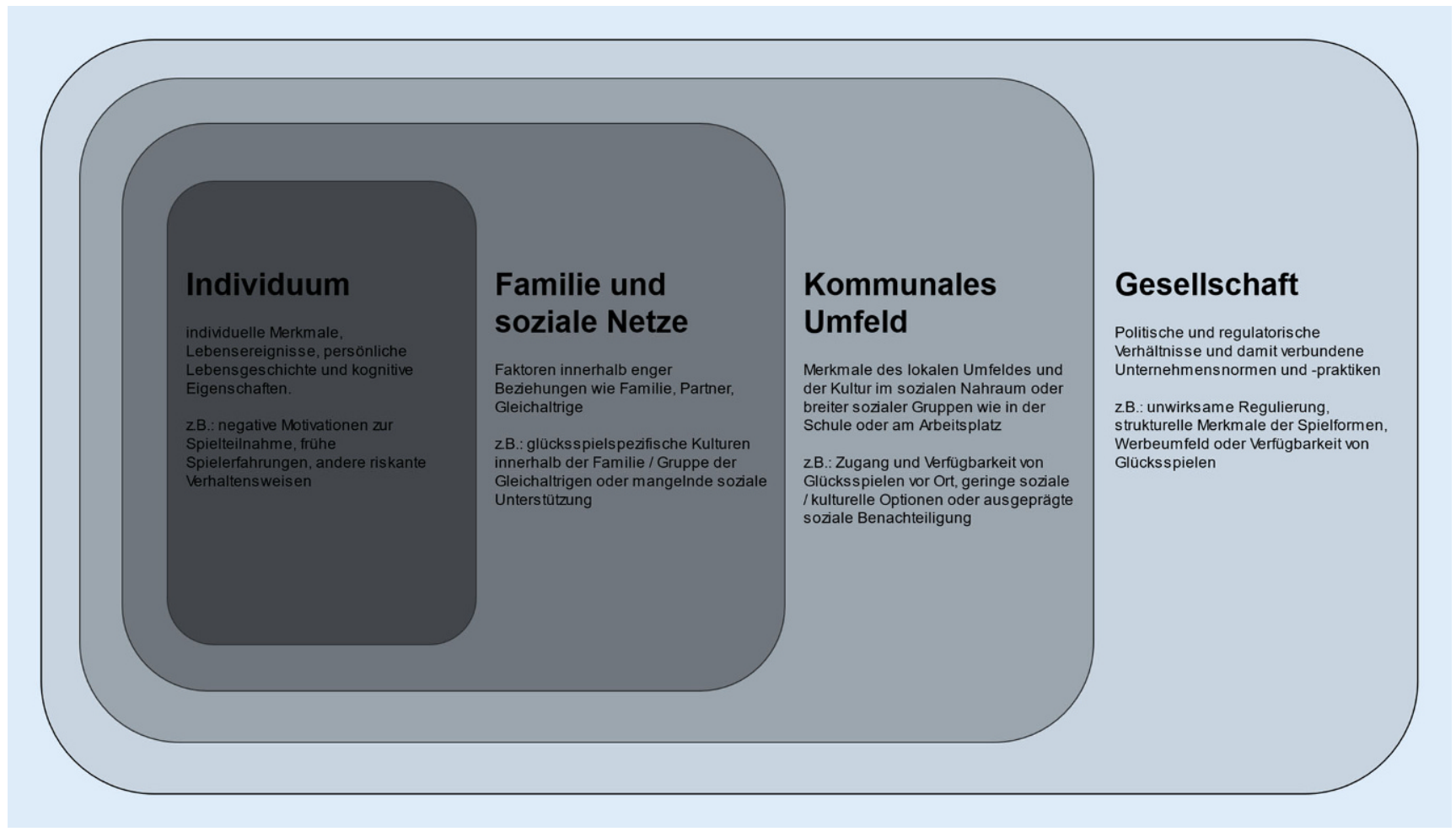

Abb. 1 ॥ Sozioökologisches Modell für glücksspielbezogene Schäden (nach [46])

Beeinträchtigungen sowie eine eigene Suchtproblematik [33].

Entsprechend breit definieren Wardle et al. [46] in ihrem Handlungsrahmen glücksspielbezogene Schäden als negative Auswirkungen des Glücksspiels auf Gesundheit und Wohlbefinden von Einzelpersonen, Familien, Gemeinden und der Gesellschaft. Diese Schäden sind vielfältig, betreffen Ressourcen, Beziehungen sowie Gesundheit und können eine Wechselwirkung von individuellen, familiären und gemeinschaftlichen Prozessen widerspiegeln. Sie können nur von kurzer Dauer sein, aber auch langfristige und dauerhafte Folgen haben, die bestehende Ungleichheiten mitunter noch verschärfen. Aus der Perspektive der öffentlichen Gesundheit bedeutet dies, dass wirksame Maßnahmen zur Schadensreduzierung nicht nur Maßnahmen zur Beeinflussung von Individuen umfassen müssten, sondern zudem Maßnahmen zur Risikominderung auf gesellschaftlicher, gemeinschaftlicher und zwischenmenschlicher Ebene (Familie und Freunde). Dieser Handlungsansatz (s. Abb. 1) basiert auf einem sozioökologischen Modell, das in anderen
Bereichen der öffentlichen Gesundheit weit verbreitet ist. Das Modell hat den Vorteil, dass es sowohl konzeptionelle Anteile hat, indem es sowohl beschreibt, wie Schaden entsteht und über mehrere Ebenen hinweg existiert als auch wie das Individuum in das soziale Gefüge eingebettet ist. Gleichzeitig erweist es sich pragmatisch, da es u.a. politischen Entscheidungsträger*innen die Festlegung ermöglicht, welche Art von Maßnahmen zur Schadensverhinderung auf jeder Ebene zu ergreifen sind. Die Autor ${ }^{*}$ innen präsentieren ferner wesentliche Kriterien für die Berechnung der sozialen Kosten auf allen Ebenen, deren Daten als wichtige Botschaft im Vorfeld weiterer Umsetzungsschritte angesehen werden (vgl. [28]).

Zukünftig sind weitere Fortschritte in der internationalen Umsetzung von Public-Health-Strategien insbesondere nach der jüngsten Entscheidung der Weltgesundheitsorganisation (WHO) zu erwarten, die die Anerkennung der glücksspielbezogenen Störung („Gambling Disorder") als Verhaltenssucht und deren Klassifikation zusammen mit substanzgebundenen Störungen in der In- ternationalen Klassifikation psychischer Störungen (ICD-11) vorsieht. Gleichzeitig hat die WHO das Glücksspiel zusammen mit Alkohol und Drogen als Thema in internationalen Foren platziert, was dessen Bedeutung als Teil einer globalen Gesundheitsfürsorge unterstreicht [4]. Im Vergleich mit anderen legalen Suchtmitteln ist das gesellschaftliche Bewusstsein über glücksspielassoziierte Gefahren zwar immer noch gering ausgeprägt. Gesundheitspolitische Erkenntnisse etwa bezogen auf Alkohol und Tabak liefern indessen wertvolle Anregungen für evidenzbasierte Handlungsstrategien für den Glücksspielbereich. Beschränkungen der Verfügbarkeit, des Zugangs und der Werbung gehören ebenso zu diesem Erfolg versprechenden Maßnahmenpaket wie eine angemessene Produktbesteuerung [29, 43, 49].

Übertragen auf den Glücksspielbereich liefert das Rahmenkonzept von Price at al. [36] eine geeignete Ausgangslage zur Implementierung einer globalen Public-Health-Strategie. Dabei werden Ansatzpunkte der Gesundheitsförderung, des Gesundheitsschutzes sowie der 
Krankheitsvorbeugung und Schadensminimierung bzw. zusätzlich aus dem Bereich der Forschung die Bestimmung der Krankheitslast auf Bevölkerungsebene sowie eine kontinuierliche Gesundheitsüberwachung (im Sinne eines Monitorings) benannt. Exemplarisch für jedes Segment sind folgende Erfolg versprechende Einzelmaßnahmen anzuführen: (1) Das Anbringen von dynamischen Warnhinweisen an Spielautomaten, die an prominenter Stelle platziert und mit regelmäßig wechselnden Botschaften auf Glücksspielrisiken oder glücksspielbedingte kognitive Verzerrungen hinweisen (Gesundheitsförderung); (2) die Umsetzung von spürbaren Verfügbarkeitsbegrenzungen und -einschränkungen v.a. bei Glücksspielen mit einem erhöhten Gefährdungspotenzial sowie in sozialstrukturell benachteiligten Settings (Gesundheitsschutz); (3) die Einführung von verbindlichen, im Vorfeld einer Spielteilnahme festzulegende Begrenzungen der Maximalspielzeit, des Maximaleinsatzes und der Maximalverluste in einem wohldefinierten Zeitfenster unter Berücksichtigung aller Glücksspielformen (Krankheitsvorbeugung und Schadensminimierung); (4) die Durchführung von repräsentativen Bevölkerungsstudien zur empirischen Abbildung des Problemausmaßes einschließlich der Bestimmung von vulnerablen Populationssegmenten und allen mit Glücksspielen assoziierten Schäden unabhängig von der Spielintensität (Krankheitslast auf Bevölkerungsebene); (5) daran anknüpfend eine stetige, systematische sowie interdisziplinäre Sammlung, Analyse und Interpretation von relevanten Gesundheits- und Evaluationsdaten (Monitoring) und deren Übersetzung in eine evidenzgestützte Programmweiterentwicklung der Public-Health-Praxis (vgl. mit [25] für die zugrunde liegenden Forschungsbefunde die ersten 3 Handlungsempfehlungen betreffend).

Insgesamt besteht der Hauptverdienst des Public-Health-Ansatzes somit zweifelsohne darin, die Perspektive auf das Krankheitsgeschehen zu erweitern und die Gesamtheit aller relevanten pathogenen Einflussfaktoren inklusive ihrer Folgen sowie deren Implikationen $\mathrm{zu}$ ihrer Vermeidung miteinzubinden. Dem- gegenüber machen Delfabbro und King [14] auf seine Grenzen im Glücksspielbereich aufmerksam: So betrifft eine Reihe von beschränkenden Maßnahmen einen großen Prozentsatz an Menschen, die auf risikoarmen Niveau spielen (und damit nicht der Kernzielgruppe angehören). Daneben werden Botschaften zur Schadensminimierungbzw. zur Bekämpfung eines problematischen Spielverhaltens von den meisten Individuen als irrelevant angesehen. Gerade die Komplexität der ursächlichen risikoerhöhenden Bedingungen auf personaler Ebene scheint daher zunächst Maßnahmen zu erfordern, die sich am Individuum orientieren (vgl. mit den Prämissen des RenoModells).

Hingegen erschweren verschiedenartige Strukturbedingungen die Entwicklung und Umsetzung einer nachhaltigen Public-Health-Policy. In erster Linie setzt ein derartig umfassender Ansatz der Gesundheitsfürsorge ein gemeinsames intersektorales Basisverständnis von Gesundheitszielen und damit die Zusammenarbeit zahlreicher Bezugsdisziplinen voraus (z. B. aus der Wissenschaft: Medizin, Psychologie, Soziologie, Gesundheitswissenschaften, Wirtschaftswissenschaften oder Kriminologie). Oftmals fußen diese Fachrichtungen allerdings auf sehr heterogenen Prämissen und $\mathrm{Pa}$ radigmen. Hinzu kommt der Föderalismus inklusive entsprechender Gesetzgebungskompetenzen: Während der Bund über die Gewerbeordnung bzw. Spielverordnung große Teile des Automatenspiels in Spielhallen und Gaststätten regelt, liegt die Hauptverantwortung für die anderen Glücksspielsegmente weitestgehend in den Händen der Bundesländer. Zudem sind es in erster Linie das Wirtschaftsministerium auf Bundesebene und die Innenressorts auf Landesebene, die den jeweiligen gesetzlichen Handlungsrahmen setzen. Hier bedarf es in Zukunft einer stärkeren Einbindung der Gesundheitspolitik als Leitdisziplin (z. B. mit der Übertragung der primären Zuständigkeit für das gewerbliche Automatenspiel auf das Gesundheitsministerium) sowie der festen Verankerung des Arbeitsgebiets der öffentlichen Gesundheit in alle Politikfelder, in denen glücksspielrelevante Entscheidungen getroffen werden.
Weiteren Vorschub für eine nachhaltige Präventionsstrategie im Sinne es PublicHealth-Ansatzes dürfte die sachgerechte Aufbereitung und Bündelung der suchtfachlichen Erkenntnisse zur Wirksamkeit von Maßnahmen des Jugendund Spielerschutzes mit sich bringen (vgl. [25]). Die interdisziplinäre und anbieterunabhängige wissenschaftliche $\mathrm{Be}$ wertung des aktuellen Forschungsstands sowie der Übertragbarkeit der empirischen Erkenntnisse auf die Verhältnisse in Deutschland sind als Leitlinien in Form von evidenzbasierten Handlungsempfehlungen festzuhalten und regelmäßig zu aktualisieren. Schließlich sollte die Einstellung der Bürger ${ }^{*}$ innen zum Glücksspiel im Allgemeinen und zur Glücksspielregulation im Speziellen ernst genommen und in Entscheidungsprozesse miteinbezogen werden. Diesbezügliche Forschungsbefunde und damit erste Anhaltspunkte lassen sich hierzulande indessen vergeblich suchen.

\section{Schlussfolgerung}

Der Vergleich der beiden Ansätze zur Schadensbegrenzung im Glücksspielbereich verdeutlicht, dass die Ausrichtung des Responsible-Gambling-Ansatzes nach dem Reno-Modell nur einen Teilaspekt des universelleren PublicHealth-Ansatzes darstellen. Während sich die vorgeschlagenen Maßnahmen eines verantwortungsbewussten Umgangs mit Glücksspielen in erster Linie an Einzelpersonen richten und Interventionen präferieren, die auf Hochrisikospieler*innen abzielen, liegt der Fokus der öffentlichen Gesundheitsfürsorge auf der Bevölkerungsebene und den Ursachen auftretender Folgeschäden. Neben den individuellen Risikofaktoren berücksichtigt dieser breitere Ansatz zielgenauer soziale, umweltbezogene und wirtschaftliche Faktoren, die zusammen mit der Identifizierung relevanter schädlicher Eigenschaften des Produkts „Glücksspiel“ als Elemente einer umfassenden Präventionsstrategie dienen können. In Anlehnung an positive Erfahrungen mit der Regulation stoffgebundener Suchtmittel werden hier insbesondere verhältnispräventive Handlungsschritte empfohlen, wie et- 
wa Eingriffe in die Spielstruktur sowie Beschränkungen der Verfügbarkeit und Werbung. Eine Verhinderung derartiger Eingriffe in das unternehmerische Handeln ist das primäre Ziel direkter politischer Einflussnahme seitens der Industrie sowie der Lobbyarbeit von Interessengruppen, um antizipierte Rückgänge der Erträge und Dividenden im Vorfeld zu blockieren. Infolgedessen erscheint der Handlungsrahmen eines verantwortungsbewussten Umgangs mit Glücksspielen für die Glücksspielanbieter als das Mittel der Wahl. Zu den Profiteuren gehören aber nicht nur private Anbieter, sondern auch der Staat, der mitunter selbst Glücksspiele veranstaltet oder erhebliche Steuereinnahmen aus diesem Bereich generiert. Somit liegt der Verdacht nahe, dass die zögerliche Haltung einiger Länder (z.B. in Großbritannien [45] oder in der Schweiz [16]), bewährte Public-HealthMaßnahmen auch im Glücksspielbereich umzusetzen, sich im Kern auf finanzielle Interessenkonflikte zurückzuführen lässt.

Ähnliche Missstände sind derzeit auch in Deutschland $\mathrm{zu}$ beobachten. Eine kritische Analyse der Vorgaben des zum 01. Juli 2021 in Kraft getretenen neuen GlüStV verweist in diesem Zusammenhang auf einen Paradigmenwechsel in der Glücksspielregulierung. Das staatliche Monopol für Glücksspiele, das eine restriktive Zulassungspraxis vorsieht, wurde durch ein Lizenzmodell ersetzt, das private Angebote kommerzieller Glücksspiele und hier insbesondere das Online-Glücksspiel mit seinen vielfältigen Facetten legalisiert. Diese weitreichende Marktöffnung dürfte mit einer deutlichen Zunahme der Verfügbarkeit von Glücksspielen sowie deren Spielanreize verbunden sein und v.a. im Online-Segment den Wettbewerb um Neukund ${ }^{\star}$ innen und Vielspieler*innen massiv befeuern [22]. Darüber hinaus entsprechen die im GlüStV verankerten Maßnahmen des Spielerschutzes weitgehend dem Handlungsrahmen eines verantwortungsbewussten Umgangs mit Glücksspielen: Offenbar legt der Gesetzgeber hier - zumindest implizit die Verantwortung in Sachen Suchtprävention größtenteils in die Hände der
Glücksspielanbieter. Unter dem Strich bedient der neue GlüStV damit vornehmlich die Interessen der Anbieter von Glücksspielen bzw. des Staates (in eindimensional-fiskalischer Hinsicht) und rückt zugleich von den aufgezeigten grundlegenden Public-HealthPrinzipien ab. Wünschenswert wäre eine alternative Gesetzgebung gewesen, die zum einen das Glücksspiel offiziell als Problem der öffentlichen Gesundheit anerkennt und zum anderen auf eine nachhaltige Implementierung evidenzbasierter Public-Health-Strategien ausgerichtet ist. Selbst erste Schritte in diese Richtung, wie die Einigung auf bundesweit einheitliche Qualitätsstandards für den Spielerschutz, die Bestimmung von Mindestanforderungen an eine wissenschaftlich fundierte sowie interdisziplinär ausgerichtete Evaluation des GlüStV oder die Aufstellung von Standards zur Vergabe von Mitteln für eine anbieterunabhängige Forschung bzw. die Konsentierung von zu priorisierenden Forschungsinhalten, sind bislang ausgeblieben. Neue Erkenntnisse in den Bereichen Forschung und Praxis sowie die - zumindest in einzelnen Ländern wahrnehmbare - zunehmende Akzeptanz derartiger Handlungsansätze wecken jedoch die Hoffnung, dass dieser Weg zukünftig auch in Deutschland beschritten wird.

\section{Fazit für die Praxis}

- Quantität und Qualität der mit kommerziellen Glücksspielangeboten verbundenen Folgeschäden verlangen nach einem effektiven Präventionsansatz. Die hierzulande unlängst erfolgte Legalisierung des OnlineGlücksspielmarkts bekräftigt diese Forderung an die Praxis nochmals.

- In der Fachliteratur werden vornehmlich zwei grundsätzlich verschiedene Strategien der Gefahrenabwehr diskutiert: Während das Reno-Modell den Fokus auf die individuelle Verantwortung der Spieler*innen legt und damit primär auf die Wirksamkeit verhaltenspräventiver Maßnahmen rekurriert, fußt das Konzept der öffentlichen Gesundheitsfürsorge (Public-Health-Ansatz) auf einem breiteren Krankheits- und Präventionsverständnis.

- Evidenzgestützte Maßnahmen wie die Reduktion der Produktverfügbarkeit, Werberestriktionen oder Eingriffe in die Veranstaltungsmerkmale sind in hinreichender Weise nur beim Public-Health-Ansatz formuliert, dessen Umsetzung im Sinne eines Good-Practice-Modells daher auch in Deutschland zu bevorzugen ist.

\section{Korrespondenzadresse}

\section{Tobias Hayer}

Studiengang Psychologie, Universität Bremen Grazerstr. 2, 28359 Bremen, Deutschland tobha@uni-bremen.de

Funding. Open Access funding enabled and organized by Projekt DEAL.

\section{Einhaltung ethischer Richtlinien}

Interessenkonflikt. G. Meyer gibt an, in den letzten fünf Jahren in hauptverantwortlicher Position finanzielle Zuwendungen in Form von Drittmitteln von verschiedenen Bundesländern und dem Rechtsausschuss des Deutschen Lotto- und Totoblockes erhalten zu haben. T. Hayer hat in den letzten fünf Jahren in hauptverantwortlicher Position finanzielle Zuwendungen in Form von Drittmitteln von dem Bundesministerium für Gesundheit, verschiedenen Bundesländern und dem Rechtsausschuss des Deutschen Lotto- und Totoblockes erhalten. Derzeit ist Herr Hayer Mitglied des Fachbeirates Glücksspielsucht nach $\S 10$ Abs. 1 Verwaltungsvereinbarung Glücksspielstaatsvertrag.

Für diesen Beitrag wurden von den Autoren keine Studien an Menschen oder Tieren durchgeführt. Für die aufgeführten Studien gelten die jeweils dort angegebenen ethischen Richtlinien.

Open Access. Dieser Artikel wird unter der Creative Commons Namensnennung 4.0 International Lizenz veröffentlicht, welche die Nutzung, Vervielfältigung, Bearbeitung, Verbreitung und Wiedergabe in jeglichem Medium und Format erlaubt, sofern Sie den/die ursprünglichen Autor(en) und die Quelle ordnungsgemäß nennen, einen Link zur Creative Commons Lizenz beifügen und angeben, ob Änderungen vorgenommen wurden.

Die in diesem Artikel enthaltenen Bilder und sonstiges Drittmaterial unterliegen ebenfalls der genannten Creative Commons Lizenz, sofern sich aus der Abbildungslegende nichts anderes ergibt. Sofern das betreffende Material nicht unter der genannten Creative Commons Lizenz steht und die betreffende Handlung nicht nach gesetzlichen Vorschriften erlaubt ist, ist für die oben aufgeführten Weiterverwendungen des Materials die Einwilligung des jeweiligen Rechteinhabers einzuholen. 
Weitere Details zur Lizenz entnehmen Sie bitte der Lizenzinformation auf http://creativecommons.org/ licenses/by/4.0/deed.de.

\section{Literatur}

1. Abbott M (2017) Gambling and gambling harm in New Zealand: A 28-year case study. Int J Ment Health Addiction 15:1231-1241

2. Abbott M (2020) Gambling control and public health: let's be really honest. Int J Ment Health Addiction 18:825-834

3. Abbott M (2020) The changing epidemiology of gambling disorder and gambling-related harm: public health implications. Public Health 184:41-45

4. Abbott M (2020) Gambling and gambling-related harm: recent World Health Organization initiatives Public Health 184:56-59

5. AbbottMW, RomildU, Volberg RA (2014) Gambling and problem gambling in Sweden: changes between 1998 and 2009. J Gambl Stud 30:985-999

6. Allami Y, Hodgins DC, Young M, Brunelle N, Currie S, Dufour M, Flores-Pajot M-C, Nadeau L (2021) A meta-analysis of problem gambling risk factors in the adult population. Addiction. https://doi.org/ 10.1111/add.15449

7. Banz M (2020) Glücksspielverhalten und Glücksspielsucht in Deutschland. Ergebnisse des Surveys 2019 und Trends. Bundeszentrale für gesundheitliche Aufklärung, Köln https://doi.org/10.17623/ BZGA:225-GS-SY19-1.0 (BZgA-Forschungsbericht)

8. Blaszczynski A, Ladouceur R, Shaffer H (2004) A science-based framework for responsible gambling: the Reno model. J Gambl Stud 20:301-317

9. Browne M, Greer N, Armstrong T, Doran C, Kinchin I, Langham E, Rockloff M (2017) The social cost of gambling to Victoria. Victorian Responsible Gambling Foundation, Melbourne

10. Browne M, Langham E, Rawat V, Greer N, Li E, Rose J, Rockloff $M$, Donaldson $P$, Thorne $H_{\text {, }}$ Goodwin B, Bryden G, Best T (2016) Assessing gambling-related harm in Victoria: a public health perspective. Victorian Responsible Gambling Foundation, Melbourne

11. Cassidy R (2014) Fair game? Producing and publishing gambling research. Int Gambl Stud 14:345-353

12. Collins P, Blaszczynski A, Ladouceur R, Shaffer HJ, Fong G, Venisse J-L (2015) Responsible gambling: conceptual considerations. Gaming Law Rev Econ 19:594-599

13. David JL, Thomas SL, Randle M, Daube M (2019) A public health advocacy approach for preventing and reducing gambling related harm. Aust N Z J Public Health 44:14-19

14. Delfabbro P, King DL (2020) On the limits and challenges of public health approaches in addressing gambling related harm. Int J Ment Health Addiction 18:844-856

15. Delfabbro PH, King DL (2021) The value of voluntary vs. mandatory responsible gambling limit-setting systems: a review of the evidence. Int Gambl Stud 21:255-271

16. Dickson C, Jeannot E, Peduzzi F, Savary JF, Costes JM, Simon O (2021) A new Swiss federal act on gambling: from missed opportunities towards a public health approach? Int J Environ Res Public Health 18(12):6575. https://doi.org/10. 3390/ijerph18126575
17. Fiedler I, Kairouz S, Reynolds J (2020) Corporate social responsibility vs. financial interests: the cas of responsible gambling programs. J Public Health 29:993-1000

18. Goudriaan AE (2014) Gambling and problem gambling in the Netherlands. Addiction 109:1066-1071

19. Hancock L, Smith G (2017) Critiquing the Reno model I-IV international influence on regulators and governments (2004-2015) — the distorted reality of "responsible gambling". Int J Ment Health Addiction 15:1151-1176

20. Hancock L, Smith G (2017) Replacing the Reno model with a robust public health approach to "responsible gambling": Hancock and Smith's response to commentaries on our original Reno model critique. Int J Ment Health Addiction 15:1209-1220

21. Hayer T (2020) Glücksspielbezogene Probleme als Public Health Aufgabe am Beispiel Jugendlicher. Public Health Forum 28:308-311

22. Hayer T (2020) Neuer Glücksspielstaatsvertrag Chancen und Risiken. Sucht Aktuell 27(2):55-59

23. Hayer T, Brosowski T, Meyer G (2020) Multi-venue exclusion program and early detection of problem gamblers: what works and what does not? Int Gambl Stud 20:556-578

24. Hayer T, Füchtenschnieder I, Hardeling A, Landgraf K, Rehbein F, Wulf R, Rumpf H-J (2020) Empfehlung zu Glücksspielwerbung während der Corona-Pandemie. Sucht66:217-222

25. Kalke J, Hayer T (2019) Expertise zur Wirksamkeit von Maßnahmen des Spieler-und Jugendschutzes: Ein systematischer Review. Peter Lang, Berlin

26. Korn DA, Shaffer HJ (1999) Gambling and the health of the public: adopting a public health perspective. J Gambl Stud 15:289-365

27. Ladouceur R, Blaszczynski A, Shaffer HJ, Fong D (2016) Extending the Reno model: responsible gambling evaluation guidelines for gambling operators, public policymakers, and regulators. Gaming Law RevEcon 20:580-586

28. Latvala T, Lintonen T, Konu A (2019) Public health effects of gambling - debate on a conceptual model. BMC Public Health 19:1077. https://doi. org/10.1186/s12889-019-7391-z

29. Livingstone $C$, Rintoul A (2020) Moving on from responsible gambling: a new discourse is needed to prevent and minimise harm from gambling. Public Health 148:107-112

30. Meyer G (2016) Der "Düsseldorfer Kreis" Lobbyismus der Glücksspielanbieter. Z Wett Glücksspielr 11:214-217

31. Meyer G (2020) Prävention der Glücksspielsucht: Warum Maßnahmen des Spielerschutzes von Glücksspielanbietern kaum Wirkung zeigen. Sucht 66:45-56

32. Meyer G (2021) Glücksspiel - Zahlen und Fakten In: Deutsche Hauptstelle für Suchtfragen (Hrsg) Jahrbuch Sucht 21. Papst, Lengerich, S119-138

33. Meyer G, Bachmann M (2017) Spielsucht Ursachen, Therapie und Prävention von glücksspielbezogenem Suchtverhalten, 4. Aufl. Springer Heidelberg

34. Meyer G, Kalke J, Hayer T (2018) The impact of supply reduction on the prevalence of gambling participation and disordered gambling behavior: a systematic review. Sucht 64:283-293

35. Newall PWS, Moodie C, Reith G, Stead M, Critchlow N, Morgan A, Dobbie F (2019) Gambling marketing from 2014 to 2018: a literature review. Curr Addict Rep 6:49-56
36. Price A, Hilbrecht M, Billi R (2021) Charting a path towards a public health approach for gambling harm prevention. J Public Health 29:37-53

37. Reynolds J, Ilacqua S, French M, Kairouz S (2020) Responsible gambling: a scoping review. Crit Gambl Stud 1:23-39

38. Rintoul A, Deblaquiere J, Thomas A (2017) Responsible gambling codes of conduct: lack of harm minimisation intervention in the context of venue self-regulation. Addict Res Theory 25:451-461

39. Schmidt-Semisch H, Thane K (2021) Moderne Drogenpolitik aus der Perspektive von Public Health. SuchtMagazin 47(3-4):12-16

40. Shaffer HJ, Blaszczynski A, Ladouceur R (2017) Truth, alternative facts, narrative, and science: what is happening to responsible gambling and gambling disorder? Int J Ment Health Addiction 15:1197-1202

41. Shaffer HJ, Ladouceur R, Blaszczynski A, Whyte K (2016) Extending the RENO model: clinical and ethical applications. Am J Orthopsychiatry 86:297-309

42. Shaffer PM, Ladouceur R, Williams PM, Wiley RC, Blaszczynski A, Shaffer HJ (2019) Gambling research and funding biases. J Gambl Stud 35:875-886

43. van der Maas $M$, Nower $L$ (2020) Contradictions of responsible gambling policies and gambling provision in the context of rapid market expansion. Gaming Law RevEcon 7:456-465

44. van Schalkwyk MC, Cassidy $R$, McKee M, Petticrew M (2019) Gambling control: in support of a public health response to gambling. Lancet 393:1680-1681

45. van Schalkwyk MCl, Petticrew $M$, Cassidy $R$, Adams P, McKee M, Reynolds J, Orford J (2021) A public health approach to gambling regulation: countering powerful influences. Lancet Public Health 6:e614-e619

46. Wardle H, Reith G, Best D, McDaid D, Platt S (2018) Measuring gambling-related harms: a framework for action. Gambling Commission, Birmingham

47. Wardle H, Reith G, Langham E, Rogers RD (2019) Gambling and public health: we need policy action to prevent harm. BMJ 365:I1807. https://doi.org/ 10.1136/bmj.I1807

48. Williams RJ, Volberg RA, Stevens RMG (2012) The population prevalence of problem gambling: methodological influences, standardized rates, jurisdictional differences, and worldwide trends. https://hdl.handle.net/10133/3068. Zugegriffen: 8. Mai 2012

49. Williams RJ, West BL, Simpson RI (2012) Prevention of problem gambling: a comprehensive review of the evidence and identified best practices. https:// hdl.handle.net/10133/3121. Zugegriffen: 1. Okt. 2012

50. Xouridas S, Jasny J, Becker T (2016) An ecological approach to electronic gambling machines and socioeconomic deprivation in Germany. J Gambl Issues 33:82-102 